\title{
An algorithm for scheduling hybrid slot-size to increase traffic density in EPONs
}

\author{
Jayanth C. ${ }^{1,}{ }^{*}$ and B. Sivakumar ${ }^{2}$ \\ ${ }^{1}$ Prist University Vallam, Thanjavur, Tamilnadu, India. \\ ${ }^{2}$ Department of Electronics and Telecommunication Engg Dr AIT, Bangalore, India.
}

Global Journal of Engineering and Technology Advances, 2021, 08(01), 028-038

Publication history: Received on 07 June 2021; revised on 09 July 2021; accepted on 11 July 2021

Article DOI: https://doi.org/10.30574/gjeta.2021.8.1.0102

\begin{abstract}
The current basically Ethernet passive optical network (EPON) efficiently supports different service classes, i.e., Giant Prime concern (GP) traffic and Efficiently-best (EE) traffic, in future-generation networks. The difficult task is finding the efficient Dynamic Bandwidth Allocation (DBA) procedure task which suggests service classes of bandwidth simultaneously. This paper suggests improved version to DBA algorithm, Remodeled version-Hybrid division-size/Rate (HDSR) scheme. The modified control message scheduling algorithm is also discussed along with Remodeled-HDSR Algorithm which fits to the proposed scheme. In the modified Remodeled-HSSR scheme, time cycle length is identical classified accordingly with two subparts. In algorithm as explained in order to increase traffic density, the subdivision part of the planned cycle, the traffic conditions are dynamically suggested an improved version for Best effort traffic of multiple ONUs.
\end{abstract}

In the First subdivision part of the span time analysis time will not be compressed, particularly at less buffer status conditions plan buffer status of the HP traffic. The Remodeled control message algorithm which predefined status and is scheduled particularly which controls connecting the sub-parts of the planned cycles of the sequence time and in-turn increases synchronization between two Gate messages to particular ONUs.

Keywords: PON; HSSR; MHSSR; Open Flow protocol; Passive Optical network (PON); Ethernet Passive Optical Network (EPON); MPCP; Water-filling-Dynamic bandwidth allocation algorithm (WF-DBA)

\section{Introduction}

Depending on details on EPON-PON, standards based on the IEEE 802.3ah [1] Ethernet which shows important task for overcoming traffic allocation blockages in wide area networks. The system based on tree networks, shown in Figure 1, consists of two subdivisions namely: the optical line terminal (OLT) which resides at the Control office (CO) and the different optical network units (ONUs) which is located at the User's home premises. The allocated frames transmission is carried out by the OLT, which passes through a 1:N passive splitter to reach particular ONU. The splitter used by passive devices which is distance located apart from the control office, but ONUs which is close to the customer home premises.

In the PON system in which bandwidth is controlled with help of message control office, i.e., Gate and Report messages, and with the help of OLT and ONUs. The Control messages studies is controlled out with the help of multi-point control protocol (MPCP) of EPON systems. The ONU of MPCP in which buffer status is updated and checked according to the OLT through a sent Report message and OLT allocated portion of the remaining upstream traffic allocation for particular ONU a Gate message is given.

\footnotetext{
${ }^{*}$ Corresponding author: Jayanth C

Department of E\&TCE Engg, Dayananda sagar college of Engineering, Bangalore, Karnataka, India. 


\section{Preliminaries}

In Ref. [1], a useful effective uplink dynamic bandwidth allocation method - demand forecasting DBA (DF-DBA) which suits ONU's advance demands by statistical modeling in order to fulfill the predicted demands, which results in reduced delay. Results show that usage of simple normal distribution in the DBA engine which offers more suitable bandwidth allocation with $14 \%$ reduced packet drop ratio (PDR) compared to values more than standard GigaPON access network (GIANT) DBA following request-grant cycle procedure.

In Ref. [2], scheme in which DBA is given accordingly for basic subdivisions of service classes of buffer status traffic, i.e., Expedited-forwarding (EF), Forward assurance (FA), and Best-Effort (BE) buffer status. In Subdivision methods, subfirst part traffic (bandwidth) allocation of bandwidth is done to the best methods traffic conditions, and then left portion of traffic is done accordingly to the BE traffic. As a part of more experimental results verified, the BE traffic which suffers more occupied delay status.

In Ref. [3], the proposed techniques to check demanding traffic conditions in which system demands and allocate more traffic depending upon conditions and reduce the dynamic loading conditions of improved based networks of the service given. In Ref. [6], hybrid slot-size/rate (HSSR) DBA algorithm is discussed accordingly in which algorithm, a time in which it is split into two equal parts. The part- 1 of the cycle is similarly divided according to the loading conditions into HP traffic of the entire ONUs while part-2 of the next cycle time is accordingly to the BE traffic of one or multiple ONUs.

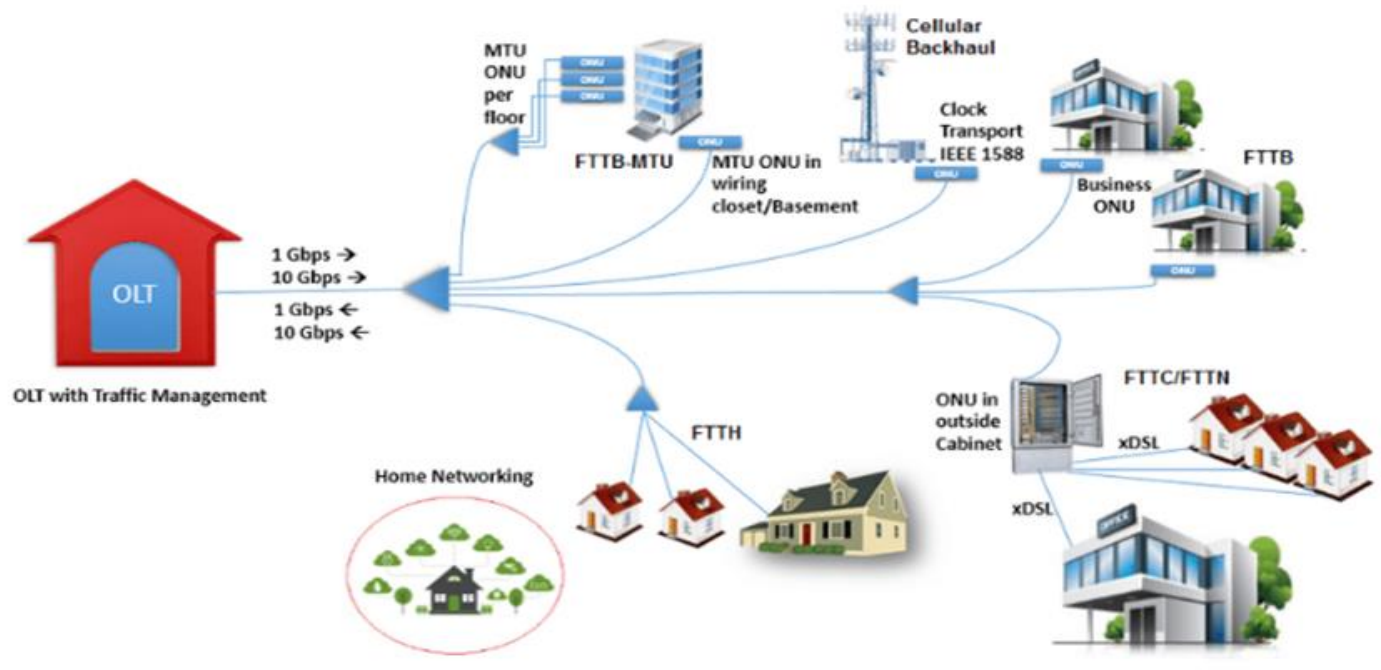

Figure 1 Next-generation Passive optical network traffic management with ONUs, OLTs

In Ref. [7], the experts (authors) have given an exposure about DBA techniques which gives all the Report messages achieved end results to the suggested window sizes to determine an ONU which requires the more allocated span length according to the allocation of particular size defined. This scheme efficiently utilizes the non-busy allocated time traffic conditions depending upon the load conditions.

A distinct efficient plan suggestion in NG-EPON is to sketch appropriate wavelength dynamically traffic is allocated in which decide future demands of the users-network. Hybrid EPON has been grouped into well defined into design architectures based on how busy traffic status is controlled on many port connections of wavelengths: SSD-EPON, MSDEPON, and wavelength agile (WA)-EPON [8]. Dynamic wavelength and traffic load conditions (DWBA-algorithm) are the well defined techniques which implement the OLT to allocate the channel bandwidth externally between amounts of wavelengths allocated which distributes for various slots basis-data communication effectively in the upstream direction. 


\section{Network architectures techniques suggested MHSSR-DBA scheme}

In the Sub-part section, first, we provide the necessary discussions about the feedbacks of the PON architecture system with the upstream format frame consisting of both HP and BE traffic. Furthermore the principle of the efficiently traffic given to the proposed MHSSR DBA scheme is discussed accordingly.

\subsection{Network Architecture of PON system}

In the preceding Fig. 2 shows suggests stock structure of the PON system with the given loading bandwidth alternations and frame layout of $N$ ONUs. The particular $N$ ONUs is given to an OLT through a passive splitter/or which combines. The each sub-division allocated packets ONU are divided accordingly into two sub grouping, i.e., HP and BE. The first top (upstream) stream division of each time cycle, the allocated bandwidth conditions are divided for HP traffic of all the ONUs is based on the given MHSSR scheme. The sub-sequent section part, the 1st sub-part of time cycle $k$ is done carried out for the HP traffic of allocatedONUs and the 2 nd sub-part of the planned cycle $k$ is carried out for best effort according to the buffer status load conditions to ONU 1 and ONU 2.

\subsection{First-FIT dynamic wavelength and allocation bandwidth algorithm}

\subsubsection{Water filling -Dynamic Bandwidth Allocation algorithm}

Water-filling algorithm [6] describes on DWBA methods (WF-DBA) which based on proceedings in [7]. A WFDBA sort the channels according to the allocation are based on top-priority basis is first given on the less loaded traffic channel, so that it uniformly suggests efficiently uniform channel.

With an sample experiments, the sub-part according to twice wavelength channels is more than the reported traffic bandwidth, method is suggested to given on only one channel; The entire concept, an ONU can be deal with more port input connections of wavelength channels which suggests upon the reported bandwidth and the contrast between the final concluding part of wavelength channels depending on entire process bandwidth allocation.

The first sub-part of WF-DBA and advanced IPACT and NG-EPON, this paper plan suggests first-fit DWBA to allocate traffic to ONUs in well distributed manner which suggest load data according to less delay and avoiding frame sequencing problems. First-fit DWBA plan gives an idea of allocation of one channel, which is given first among all the channels at the particular instant of time. References [8-11] use a well defined plan to suggest an plan for multithread polling in reach PONs.

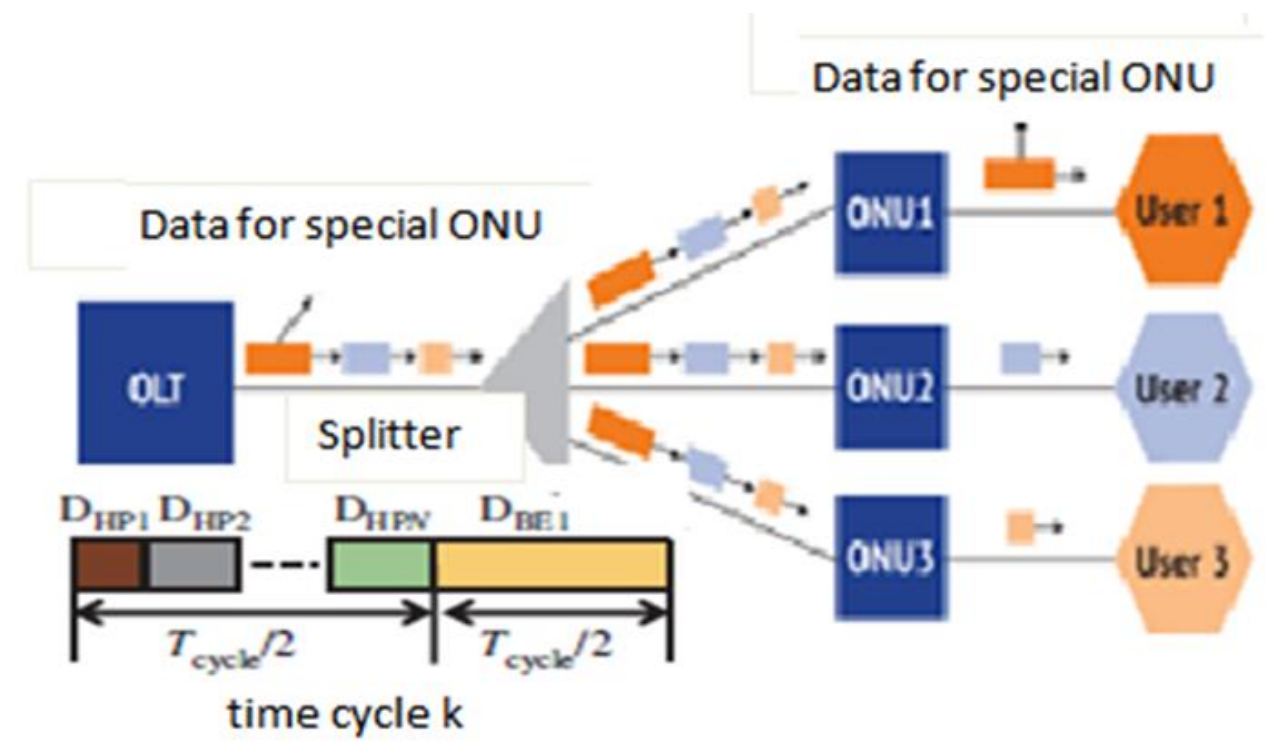

Figure 2 PON-upstream frame format of the proposed system architecture 


\subsection{First-FIT DWBA scheme}

Analysis is carried out many times of WF-DBA and modified-IPACT and advancements is carried out for NG-EPON, this scheme suggested method of First-Fit method of DWBA to allocated traffic depending upon loading (buffer) conditions to ONUs in a disciplined manner data buffer display and reschedules frame according to loading conditions. First-fit method of DWBA grants on each channel and it will be suitable at the time of suggested decision. References [8-11] deals an important method for multithread polling in Longer range of PONs, the efficient method, no such problems has been given to address.

The following symbols are used in the suggested algorithm:

\begin{tabular}{|c|l|}
\hline $\mathbf{O N U}_{\mathbf{i}}$ & $\mathbf{i = 1 , . .}$ up to N: No of ONUs allocated in network \\
\hline $\mathrm{RTT}_{\mathrm{i}}$ & Between ONUi and OLT- Round-trip propagation time varies \\
\hline $\mathrm{W}$ & No of upstream channels -wavelengths allocated for the network, $\left\{\lambda_{1} ; \lambda_{2} ; \lambda_{3} ; \lambda_{4}\right\} ;$ \\
\hline $\mathrm{T}_{\mathrm{c}}$ & Allocation time for communication of control frame guard time; \\
\hline $\mathrm{G}_{\mathrm{i}}$ & Bandwidth is reported to the $\mathrm{i}^{\text {th }}$ ONU; \\
\hline$\left\{\begin{array}{c}k \\
(n, s)\end{array}\right\}$ & $\begin{array}{l}\text { Allocation is done based on start time of channel of last bit-(nth) ONU during which } \\
\mathrm{s}^{\text {th }} \text { transmission is scheduled on wavelength channel k; }\end{array}$ \\
\hline $\begin{array}{c}k \\
(n, s)\end{array}$ & $\begin{array}{l}\text { scheduled time completed for allocation of } \mathrm{n}^{\text {th }} \text { ONU accordingly } \text { sh }^{\text {th }} \text { scheduled traffic } \\
\text { load conditions on wavelength channel } \mathrm{k},\end{array}$ \\
\hline
\end{tabular}

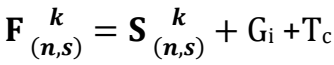

$\mathrm{R}_{\mathrm{t}}$ : Report plan time of ONUi at OLT .

\section{First-Fit DWBA tasks scheduled}

- $\mathrm{s}^{\text {th }}$ scheduled pattern depends on ONUi; allocated bandwidth-reported bandwidth $\left(\mathrm{G}_{\mathrm{i}}\right)$

- In ascending order sort wavelengths set W depending on their start time

- Grants channel based on first-fit depending on transmission at that time where $\chi_{\mathrm{k}}=\arg _{\mathrm{k}} \min \left\{\mathrm{F}_{(n-1, s)}^{k}\right\}$ where $\lambda_{\mathrm{k}} \varepsilon$ (belongs to) $\mathrm{W}$

- $\quad$ Allocated start time is decided based on

- Updated completion time on wavelength $\mathrm{k}$,

$$
\mathrm{S}_{(n, s)}^{k}=\max \left(\lambda \mathrm{k}, \mathrm{t}+\mathrm{RTT}_{\mathrm{s}}\right)
$$

$$
\mathrm{F}_{(n, s)}^{k}=\mathrm{S}_{(n, s)}^{k}+T_{\mathrm{C}}+G_{\mathrm{i}}
$$

\subsection{Suggested method DBA-MHSSR Scheme}

The first part which describes MHSSR scheme, the span of cycle is primarily ranged into two sub-divided parts, which is indistinguishable to the HSSR and DVGP strategies. Initially, the 1st sub-part of cycle is accordingly divided and it is used for the HP traffic while the part-2 accordingly given and is used for the Long reach buffer status of the ONUs in the predefined PON techniques. The subsequent equation which defines the span length calculation of a time cycle $T$ cycle is suggested below.

The maximum allocated length of time cycle $T_{\text {cycle }}^{\max }$ is calculated as follows:

$T_{\text {cycle }}^{\max }=N\left[\mathrm{~W}_{(H P)}^{\max }+\mathrm{Tg}\right]+N\left[\mathrm{~W}_{(B E)}^{\max }+\mathrm{Tg}\right]$

The ONUs, $L$ which specifies traffic allocation ONUs being coordinates to more traffic loads in a time cycle, $W_{H P}^{\max }$ and $W_{B E}^{\max }$ are the given in particular window sizes for the HP and BE traffic of $\mathrm{ONU}_{\mathrm{i}}$, commonly, and $\mathrm{Tg}$ is the guard time. 
Where $\mathrm{W}_{(H P)}^{\max }$ and $\mathrm{W}_{(B E)}^{\max }$ are the efficient window sizes which depends on HP and BE traffic, and in the preceding method, the next method consider

$$
\mathrm{W}_{(H P)}^{\max }=\mathrm{W}_{(B E)}^{\max }=\frac{\left[T_{\text {cycle }}^{\max }-2 N \mathrm{Tg}\right]}{2 n}
$$

The involvement of vitally allocation, the proposed scheme which separates the non occupied predefined slots as well as fewer-load buffer conditions cost effective increased bandwidth from the buffer status fewer loaded ONUs to depending upon its ONUs. Fig.3 suggests the efficient plan of the PON system using given MHSSR DBA system.

In the Fig. 2, sub-division parts i.e., time cycles $k$ and $k+1$, are suggested according buffers status of traffic grants scheme. Accordingly the 1st sub-part cycle $k$, more bandwidth $\mathrm{W}_{(B E)}^{N}$ (HP) is taken from little loaded ONUs with W ${ }_{(H P)}^{\max }$ is given for the ONU $N$. In the 1st sub-part of span of cycle $k+1$, the complete ONUs is more loaded. With verifying the result analysis the allocated bandwidths (buffer status) is carried out ONU1, ONU2, and ONU3........NU.

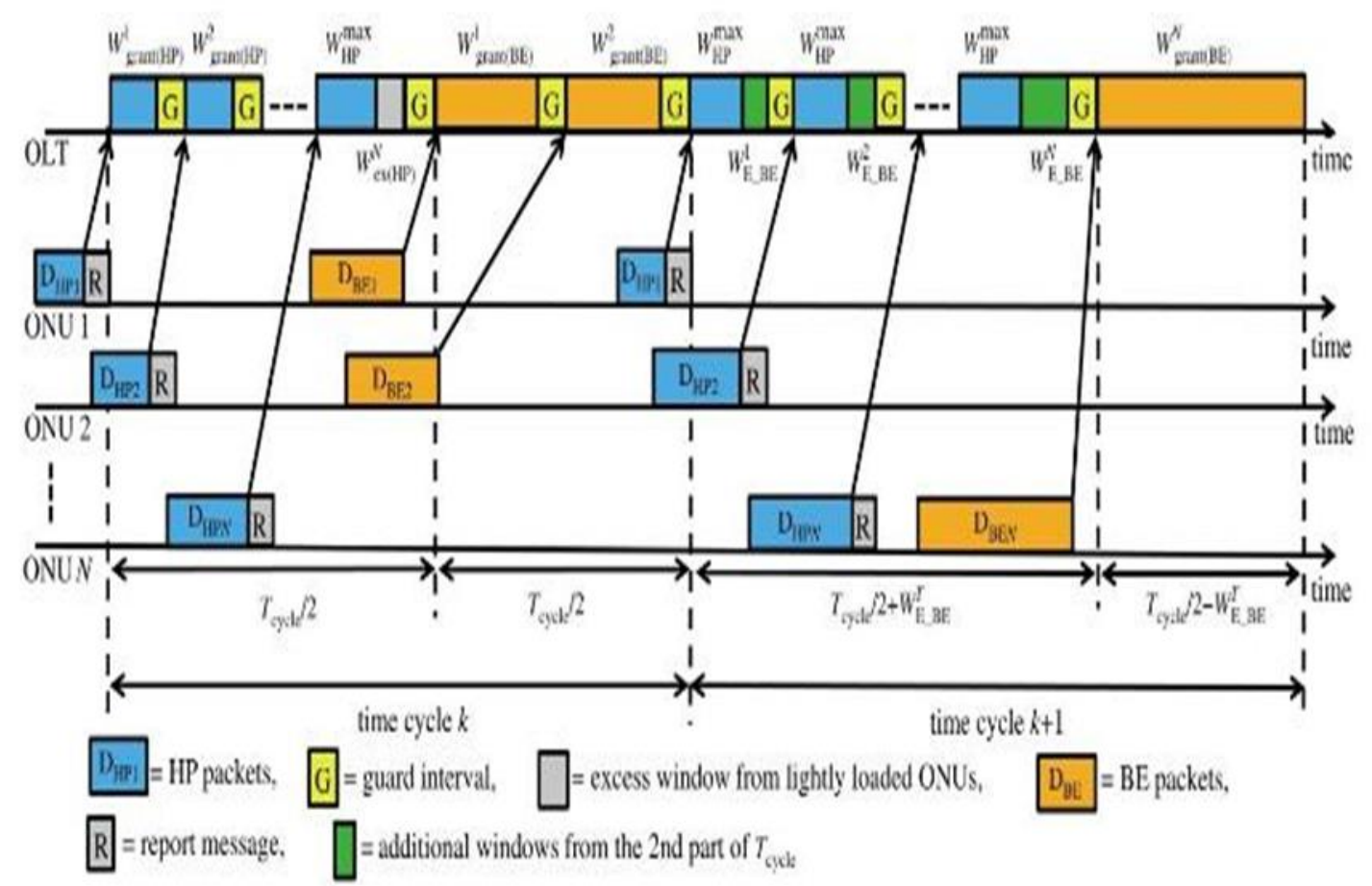

Figure 3 Allocation of bandwidth for upstream in the Proposed Remodeled HSSR Dynamically Bandwidth Allocation (DBA) scheme

$\mathrm{W}_{\mathrm{ex}(H P)}^{i}$ for any ONU I allocation is not carried out which suggests, the traffic buffer status for different ONU 1, ONU $2, \ldots . .$. upto entire $\mathrm{ONU} N$ i.e., $\mathrm{W}_{\text {grant }(H P)}^{1}, \mathrm{~W}_{\operatorname{grant}(H P)}^{2} \ldots \ldots \ldots$ upto entire. $\mathrm{W}_{\operatorname{grant}(H P)}^{n}$ consists of $\mathrm{W}_{(H P)}^{\max } \quad \mathrm{plus}^{2}$ $\mathrm{W}_{\mathrm{E}_{-} B E}^{1}, \mathrm{~W}_{\mathrm{E}_{-} B E}^{2} \ldots \ldots \ldots \ldots \ldots \mathrm{W}_{\mathrm{E}_{-} B E}^{\mathrm{N}}$ respectively. Here, $\mathrm{W}_{\mathrm{E}_{-} B E}^{1}, \mathrm{~W}_{\mathrm{E}_{-} B E}^{2} \ldots \ldots \ldots \ldots . \mathrm{W}_{\mathrm{E}_{-} B E}^{\mathrm{N}}$ are the bandwidth from the $2^{\text {nd }}$ sub-part of plan rotation cycles $\mathrm{k}+1$ for $\mathrm{ONU} 1$, ONU 2, . ONU $N$, commonly. The entire allocated buffer status of bandwidth from the part- 2 cycle $k+1, \mathrm{~W}_{\mathrm{E}_{-}(B E)}^{T}$ is which examines $\mathrm{W}_{\mathrm{E}_{-} B E}^{1}, \mathrm{~W}_{\mathrm{E}_{-} B E}^{2} \ldots \ldots \ldots \ldots \ldots \mathrm{W}_{\mathrm{E}_{-} B E}$. As a result, the preceding cycle $k+1$ is $\mathrm{T}_{\text {cycle/2 }}+\mathrm{W}_{\mathrm{E}_{-} B E}^{\mathrm{T}}$ and the left portion of part- 2 of time cycle $k+1$ is gradually reduce depending on $\mathrm{T}_{\text {cycle/2 }}-\mathrm{W}_{\mathrm{E}_{-} B E} \mathrm{~T}$

Case 1 The entire ONU is accordingly to HP traffic is given loading bandwidth conditions. In this case, Eq. (3) which calculates allocated traffic for complete traffic conditions $\mathrm{W}_{\operatorname{grant}(H P)}^{i}$ of $\mathrm{ONU}_{\mathrm{i}}$.

$\mathrm{W}_{\operatorname{grant}(H P)}^{i}=\mathrm{W}_{\mathrm{R}(H P)}^{i}$ ie., $\mathrm{W}_{\mathrm{R}(H P)}^{i} \leq \mathrm{W}_{(H P)}^{\max }$ 
Case 2 Here, less ONUs are loaded traffic is fewer and little ONUs are given additional traffic, but the whole required window allocated sizes for the more traffic of preceding ONUs is more or less equal to the equation given below.

$$
\frac{T_{\text {cycle }}}{2} \text {, ie. } \sum_{\mathrm{i}=1}^{N} \mathrm{~W} \underset{\mathrm{R}(H P)}{\mathrm{i}} \leq \frac{T_{\text {cycle }}}{2}
$$

This condition mainly suggests in the cycle $k$ of the Fig. 3. In this case, it suggest to calculate $\mathrm{W}_{\operatorname{grant}(H P)}^{i}$

$$
\begin{gathered}
\mathrm{W}_{\operatorname{grant}(H P)}^{i}=\left(\mathrm{W}_{\mathrm{R}(H P)}^{i}\right) \text { if } \mathrm{W}_{\mathrm{R}(H P)}^{i} \leq \mathrm{W}_{(H P)}^{\max } \\
\left(\mathrm{W}_{(H P)}^{\max }+\mathrm{W}_{\operatorname{ex(HP)}}^{i}\right) i f \mathrm{~W}_{\mathrm{R}(H P)}^{i}>W_{(H P)}^{\max } \cdots \cdots .
\end{gathered}
$$

Where $\underset{\operatorname{ex}(H P)}{i}$ is the more bandwidth given for the highly allocated traffic of ONU $i$.

The preceding qu (5) is used to calculateW $\underset{\operatorname{ex}(H P)}{\mathrm{T}}$.

$$
\mathrm{W}_{\operatorname{grant}(H P)}^{T}=, \sum_{\mathrm{i}=1}^{N}\left(\mathrm{~W}_{\mathrm{R}(H P)}^{\max }-\mathrm{W}_{\mathrm{R}(H P)}^{\mathrm{i}}\right), \ldots \ldots
$$

Which defined in which $\mathrm{M}$ is the no of equally less full-filled ONUs in a given plan cycle.

The preceding, Eq. (6) which is used to calculate where the bandwidth is distributed fairly this $\mathrm{W}_{\operatorname{ex}(H P)}^{T}$ to more allocated traffic to $\mathrm{ONU}_{i}$.

$$
\mathrm{W}_{\operatorname{ex}(H P)}^{\mathrm{i}}=\frac{\mathrm{W}_{\operatorname{ex}(H P)}^{T} \times \mathrm{W}_{\mathrm{R}(H P)}^{i}}{\sum_{i=1}^{x} \mathrm{~W}_{\mathrm{R}(H P)}^{i}}
$$

Which defines accordingly Xis the no's of given traffic ONUs in a particular allocated plan cycle $\mathrm{W}_{\mathrm{E}_{-} \mathrm{BE}}^{\mathrm{T}}$ is remaining (left) bandwidth from the part-2 of Tcycle. Eq. (7) in preceding equation calculates $\mathrm{W}_{\mathrm{E}_{-} \mathrm{BE}}^{\mathrm{T}}$

$$
\mathrm{W}_{\mathrm{E} \_\mathrm{BE}}^{\mathrm{T}}=\sum_{i=1}^{\mathrm{N}} \mathrm{W}_{\mathrm{R}(H P)}^{i}-\frac{\mathrm{T}_{\text {cycle }}}{2} .
$$

Case 3 In next step conditions, depending on ONUs are given more bandwidth load conditions and the sum of the equally allocated windows of the more traffic of all the

ONUs is greater than $\mathrm{T}_{\text {Cycle }} / 2$, i.e.,

$$
\begin{gathered}
\sum_{i=1}^{\mathrm{N}} \mathrm{W}_{\mathrm{R}(H P)}^{i}>\mathrm{T}_{\frac{\text { cycle }}{2}}^{i} \text { and } \\
\sum_{i=1}^{\mathrm{N}} \mathrm{W}_{\mathrm{R}(H P)}^{i}-\mathrm{T}_{\text {cycle } / 2}=\mathrm{W}_{\mathrm{E} \_\mathrm{BE}}^{\mathrm{T}} \cdot .
\end{gathered}
$$


This way method analysis of results is carried out in time cycle $k+1$ in Fig. $3 . \mathrm{W}_{\operatorname{grant}(\mathrm{HP})}^{\mathrm{i}}$ is used to given the analysis based on time cycles.

$$
\mathrm{W}_{\operatorname{grant}(\mathrm{HP})}^{\mathrm{i}}=\mathrm{W}_{(H P)}^{\max }+\mathrm{W}_{\mathrm{E} \_\mathrm{BE}(3)}^{i}
$$

Fig. 4 below defines the flow-diagram of the given method of DBA-MHSSR process. In each time cycle, the ONUs is given accordingly, and then $\mathrm{W}_{(H P)}^{\max }$ and process are carried accordingly. After the results MHSSR DBA scheme is given some important suggestions based on $N$ ONUs in organization network, where part-3 cases of the MHSSR given plan are given accordingly to $\mathrm{ONU}$.

\subsection{Progress evaluation and experimental software procedures}

In this section, evaluation results, parameters results, and of the MHSSR scheme are tabulated accordingly to the needs and verified results depends on traffic load conditions. Repeated conduction with various analysis of experiments with different conditions were check the results of suggested DWBA with respect to modified-IPACT and WF-DBA with $\lambda_{\max }=4$ and $\lambda_{\max }=2$.

Table 1 SOFTWARE SIMULATION PARAMETERS

\begin{tabular}{|l|l|l|}
\hline \multicolumn{1}{|c|}{ Notations } & \multicolumn{1}{|c|}{ Explanations } & \multicolumn{1}{c|}{ service } \\
\hline$N$ & ONUs Numbers & 16 \\
\hline$n_{\mathrm{B}}$ & Buffers allocated & 2 \\
\hline$n_{P}$ & Packet classes Numbers & 2 \\
\hline$T_{\max }$ & More length given according to cycle & $2 \mathrm{~ms}$ \\
\hline$L$ & $\begin{array}{l}\text { Criteria based on OLT and ONUs } \\
\text { distances are varied }\end{array}$ & Range 10 to $20 \mathrm{~km}$ \\
$T_{g}$ & Guard time & $508 \mathrm{~s}$ \\
\hline$R$ & $\begin{array}{l}\text { Data rate } \\
\text { Ethernet expense span }\end{array}$ & $1 \mathrm{Gbit} / \mathrm{s}$ \\
$T_{\mathrm{EO}}$ & \multicolumn{2}{|c|}{$576 \mathrm{bits}$} \\
\hline
\end{tabular}

Fig. 5 shows the midpoint terminal to terminal ends bundle delays vs. offered buffer loads for the Best effort traffic conditions of the given MHSSR and surviving DVGP and HSSR techniques.

Fig.6 shows the percentage bandwidth allocated remains unallocated wastage for particular lengths of cycle's ie., $0.5 \mathrm{~ms}$ to $3 \mathrm{~ms}$. The techniques so far discussed according to DVGP and HSSR provide bandwidth wastage which is still pending than the plan proposed in this paper. The allocation of bandwidth for the HP traffic in both techniques is not changed for load which is allocated and also causes bandwidth idle for the less loaded ONUs. In contrast our Remodeled HSSR scheme bandwidth is improved for the HP traffic where idle bandwidth can be reduced in each ONU. 


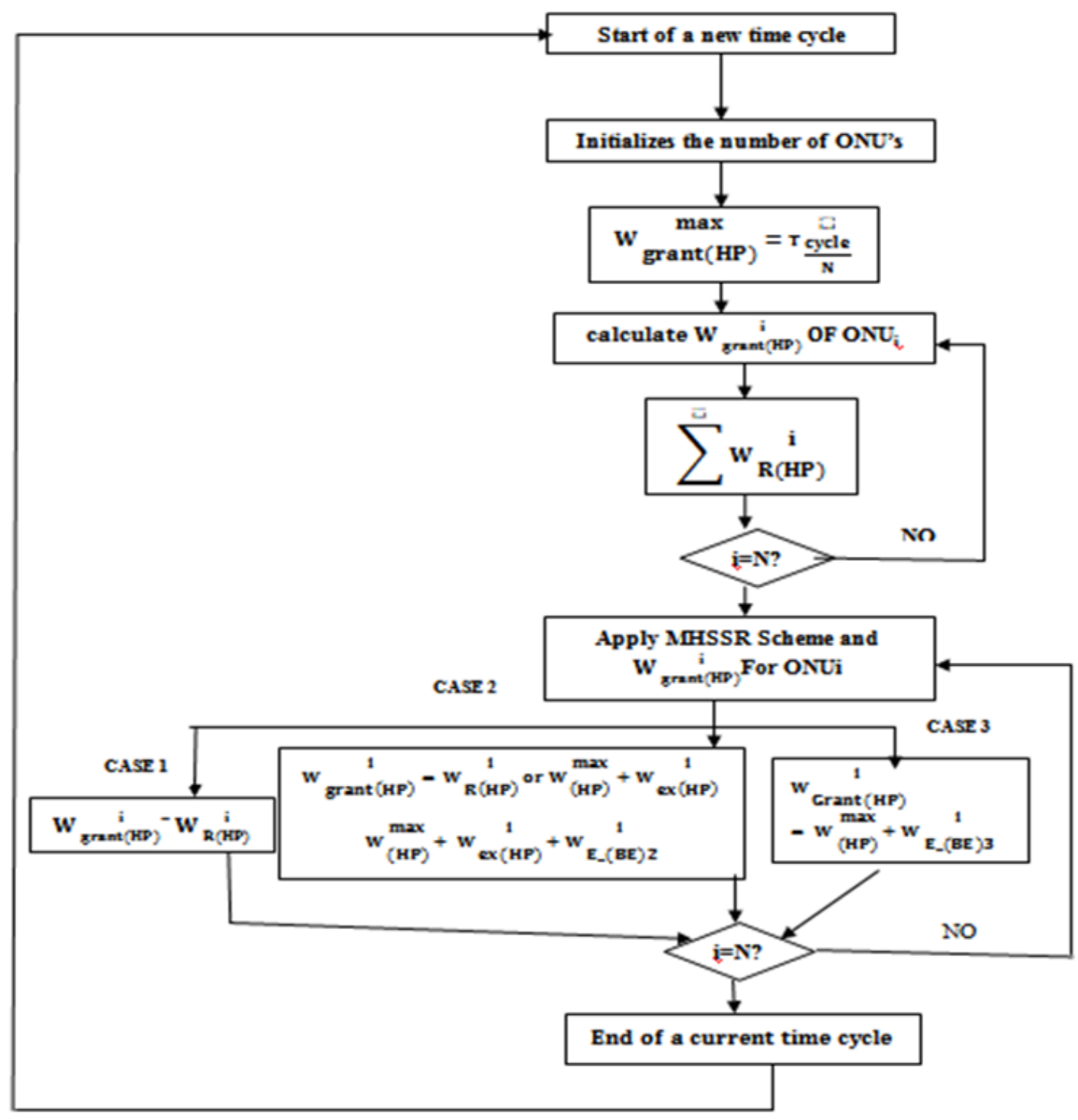

Figure 4 Proposed system- Flow chart of MHSSR Dynamic bandwidth allocation (DBA) scheme

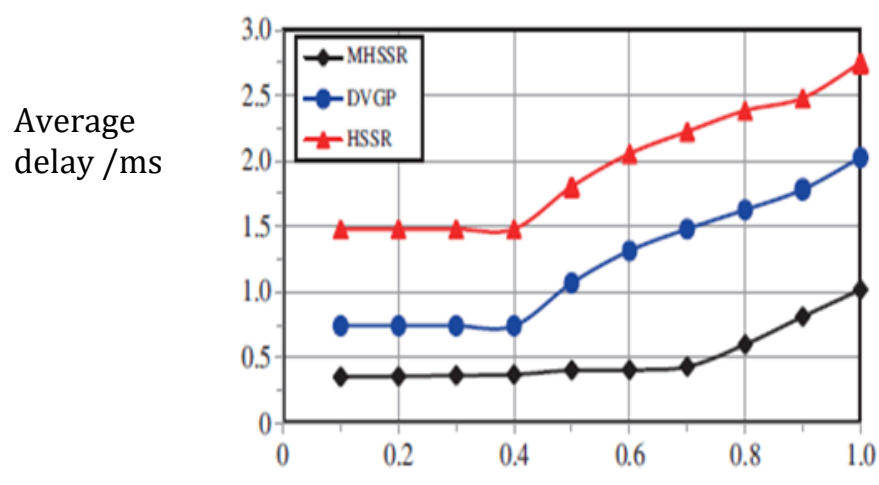

Offered load

Figure 5 Terminal ends Average Packet delay vs. different loads depending on 2ms time cycle 


\section{Wastage bandwidth Percentage}

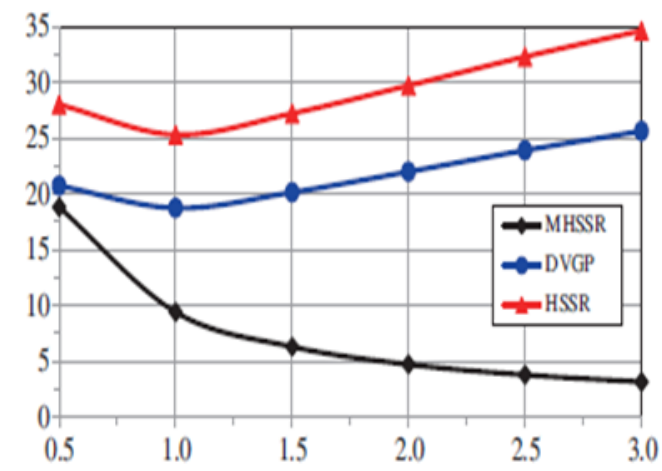

Maximal cycle time/ms

Figure 6Bandwidth reduction comparison for distinct cycle process

Table 2 Observed test results parameters

\begin{tabular}{|l|c|}
\hline \multicolumn{1}{|c|}{ Criteria } & Assessment \\
\hline Wavelengths no's allocated & 4 \\
Data rate allocated for wavelength & $25 \mathrm{Gbps}$ \\
\hline ONUs Allocation & 128 \\
Buffer allocated for each ONU & 10 Mbytes \\
\hline Distribution depends on size of Ethernet packet & $64-1.518 \mathrm{M}$ bytes \\
Control message (MPCP) & 64 bytes \\
\hline Upstream in which Inter-frame gap & 12 bytes \\
Frame preamble & 8 bytes \\
\hline Guard time & $1 \mu \mathrm{s}$ \\
RTT & $100 \mu \mathrm{s}$ \\
\hline
\end{tabular}

Average delay (ms)

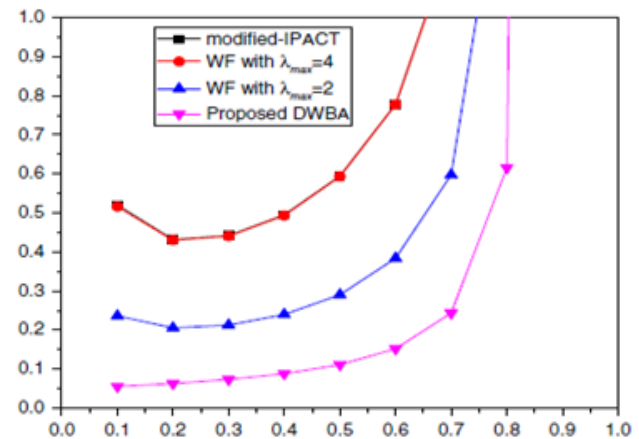

Normalized load

Figure 7 Packet delay median-average analysis of Improved-IPACT, WF-Dynamically bandwidth algorithm, and plan given to DWBA

Fig.7 represents the Plan suggested to Improve-IPACT, WF-DBA and suggested accordingly for DWBA by contrast and gives traffic midpoint packet delay at particular load. The Frames depends on less allocated conditions with respect to fewer loads depending on home user's conditions. 


\section{Conclusion}

This paper illustrates First-fit DWBA strategy defining EPON for future generation networks in order to grant less bandwidth for transmission and to cause fewer problems for re-sequencing by allocating ONU data frames on a wavelength channel which will be one grant allocation. Here method suggests bandwidth efficiently utilized by overcoming problems guard time contrast with WF-DBA, rebuilt-IPACT DBA. ONU is allowed to communicate data which is equal to bandwidth outline on one particular channel allocated and which reduce bandwidth usage in terms of granted time, frames which is denied for transmission has been left out and bandwidth remaining in which it happens frame size mismatch in which of Remodeled-IPACT and WF-DBA.

In this paper it improves a version of DBA Algorithm the Remodeled-HSSR scheme which increases stability in high speed traffic in EPON system.

\section{Compliance with ethical standards}

\section{Acknowledgments}

The author would like to thank Dr. B. Sivakumar, Professor, Dept of Electronics and Telecommunication Engg, Dr AIT, Bangalore for helping me in order to publish this article.

\section{Disclosure of conflict of interest}

The author declares no conflict of interest.

Statement of ethical approval

\section{References}

[1] Kamran A. Memon, Khalid H. Mohammadani, Asif A. Laghari, Rahul Yadav. Dynamic Bandwidth allocation algorithm with demand forecasting mechanism for bandwidth allocation in 10 Gigabit capable Passive Optical Networks, Optik, International Journal for Light and Electron optics. March 2019.

[2] Mohammad Mahbubur, Rahman, Monir Hossen. Bandwidth Allocation and Control Message Scheduling Algorithms for Improving the QoSs of High Priority Traffic in PON, Journal of Communications and Information Networks. 2018; 3(4).

[3] RL Graham. Bounds for certain multiprocessing anomalies, Bell Syst. Tech. J., vol. 45, no. 9, pp. $1563-1581,1966$. Hussain et al. VOL. 9, NO. 12/DECEMBER 2017/J. OPT. COMMUN. NETW. 1115.

[4] Bo Liu, Lijia Zhang, Fu Wang, Ming Liu, Yaya Mao. Adaptive dynamic wavelength and bandwidth allocation algorithm based on error-back-propagation neural network prediction, Journal of Optical Communications and Networking, Received in revised form 21 November 2018; Accepted 17 December 2018.

[5] Syed Baqar Hussain, Haiyun Xin, Weisheng Hu, Ahmed Mohammed Mikaeil. Low-Latency Dynamic Wavelength and Bandwidth Allocation Algorithm for NG-EPON, Journal of Optical Communications and Networking. December 2017.

[6] G Kramer, B Mukherjee, G Pesavento. Ethernet PON (ePON): Design and analysis of an optical access network, Photon. Netw. Commun.2001; 3(3): 307-319.

[7] G Kramer, G Pesavento. Ethernet passive optical network (EPON): Building a next-generation optical access network, IEEE Commun. Mag. 2002;40(2): 66-73.

[8] https://www.cisco.com/c/dam/en/us/solutions/collateral/serviceprovider/visual-networking-indexvni/complete-white-paperc11- 481360.pdf.

[9] W Hou, Z Ning, L Guo, et al. Service degradability supported by forecasting system in optical data center networks [J]. IEEE System Journal. 2018.

[10] G Kramer. A proposal for channel bonding at MAC control sublayer, in IEEE 802.3ca 100G-EPON Task Force. 2016. 


\section{Author's short biography}

Jayanth C., Reserarch scholar, Prist university,Vallam, Thanjavur, Tamilnadu working
as a Assistant Professor of Electronics and Telecommunication, Dayananda Sagar
College of Engineering, Bangalore since March 2008. My current research interests
include Optical networks, Wireless Networks and Antenna Measurements
communications in general and quality of service related to optical Networks and
protocols in particular.

